\title{
Promotion of Form I' in the Polymorph Selection of Polybutene-1 During Crystallization under High Gas/Supercritical Fluid Pressure via Enhancing Chain Mobility
}

Yongna Qiao, ${ }^{a, b}$ Jing Liu, ${ }^{b}$ Jinchuan Zhao, ${ }^{b}$ Menglong Xu, ${ }^{b}$ Qing Qii, ${ }^{b}$ Zuolong Chen, ${ }^{a}$ Yongfeng Men, ${ }^{c}$ Chul B. Park, ${ }^{b, *}$ Patrick C. Lee ${ }^{a, *}$

a. Multifunctional Composites Manufacturing Laboratory, Department of Mechanical and Industrial Engineering, University of Toronto, 5 King's College Road, Toronto M5S 3G8, Canada.

b. Microcellular Plastics Manufacturing Laboratory, Department of Mechanical and Industrial Engineering, University of Toronto, 5 King's College Road, Toronto M5S 3G8, Canada.

c. State Key Laboratory of Polymer Physics and Chemistry, Changchun Institute of Applied Chemistry, Chinese Academy of Sciences, Renmin Street 5625, Changchun 130022, P. R. China.

*Email: patricklee@mie.utoronto.ca (P.L.); park@mie.utoronto.ca (C.P.) 

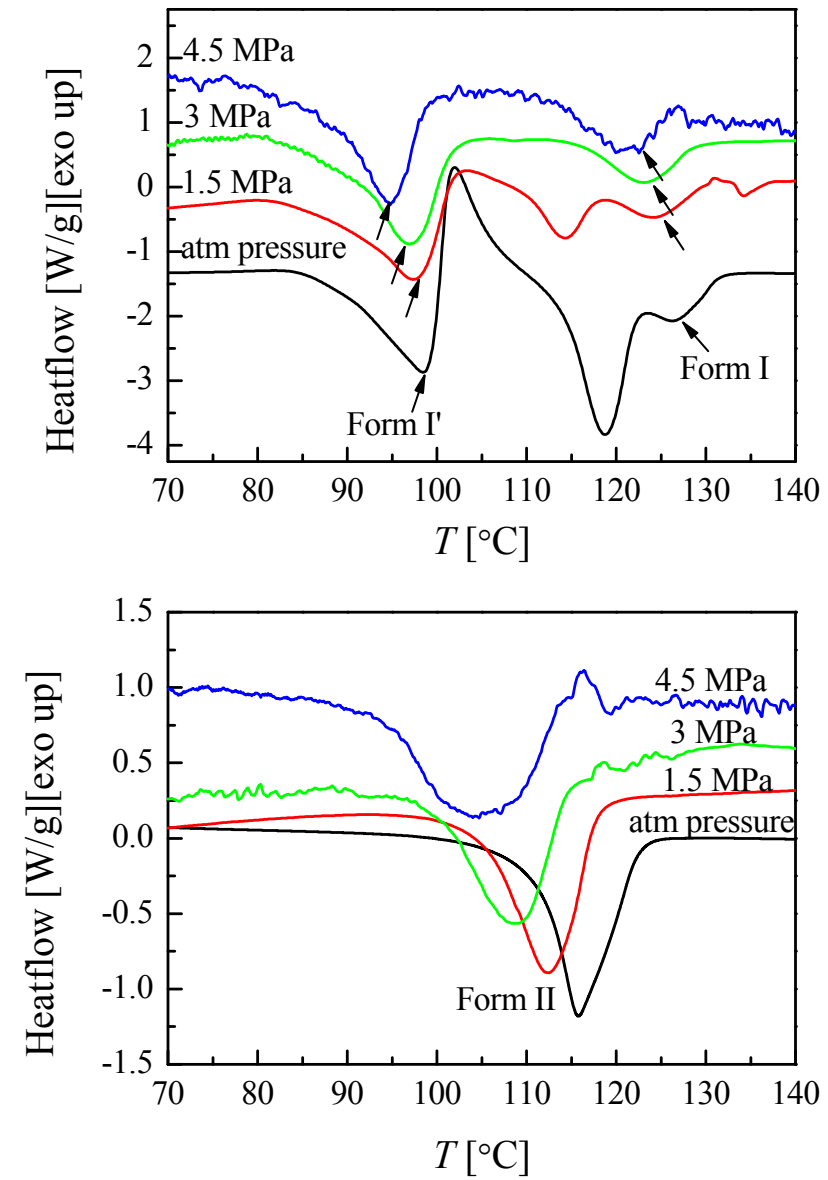

Figure S1. The melting curves of PB-1 in form I/I' (top) and in form II (bottom) measured by high-pressure DSC as a function of $\mathrm{CO}_{2}$ pressure. 